\title{
Search and Social Integration
}

\author{
Edward Y. Chang \\ Google Research China \\ edchang@google.com
}

\begin{abstract}
Search and Social have been widely considered to be two separate applications. Indeed, most people use search engines and visit social sites to conduct vastly different activities. This talk presents opportunities where search and social can be integrated synergistically. For instance, on the one hand, a search engine can mine search history data to facilitate uses to engage in social activities. On the other hand, user activities at social sites can provide information for search engines to improve personalized targeting. This talk uses Confucius, a Q\&A system which Google develops and has launched in more than 60 countries, to illustrate how computer algorithms can assist synergistic integration between search and social. Algorithmic issues in data mining, information ranking, and system scalability are discussed.
\end{abstract}

